\title{
Current Status of Drug-Eluting Stents and Drug-Eluting Balloons for the Superficial Femoral Artery
}

\author{
Joy P. Walker • Christopher D. Owens
}

Published online: 6 April 2013

(c) Springer Science + Business Media New York 2013

\begin{abstract}
The endovascular management of symptomatic atherosclerotic superficial femoral artery disease is challenging and requires consideration of unique anatomic, hemodynamic, and biomechanical factors. For innovative local drug delivery technologies to have a cost-effective and clinically meaningful benefit, they must provide patency rates in more complex lesions equivalent or superior to those currently approved devices are able to provide. Several proof-of-concept trials have either been published or been recently presented and many more are in the pipeline suggesting biologic effectiveness of these hybrid devices in reducing restenosis. Local drug delivery technology has already been commercially introduced in some countries for a variety of clinical settings. However, although these technologies offer promise in improving outcomes following lower extremity intervention, caution and safety are paramount. Adequately powered, multicenter, well-designed, randomized controlled trials with long-term follow-up (3-5 years) are still needed to accurately assess safety and efficacy.
\end{abstract}

Keywords Femoral artery - Drug-eluting stent . Drug-coated balloon - Local drug delivery .

Superficial femoral artery

\section{Introduction}

One of the of the current challenges to the endovascular management of lower extremity atherosclerotic occlusive

J. P. Walker · C. D. Owens ( $\square)$

Division of Vascular and Endovascular Surgery, University

of California, San Francisco, 400 Parnassus Ave. A581,

San Francisco, CA 94143, USA

e-mail: christopher.owens@ucsfmedctr.org disease is establishing better patency rates in more complex lesions. To date, most trials involve relatively short lesions within the superficial femoral artery (SFA) in patients with claudication, Rutherford category 1-3 disease. Long total occlusions, heavily calcified arteries, and stenosis in diabetic patients located in areas of high mechanical stress, i.e., the distal superficial and popliteal arteries, remain major hurdles for which effective solutions remain elusive.

Benchmark investigational device exemption trials have demonstrated respectable 12-month primary patency rates for bare metal stents (BMS) in lesions less than $10 \mathrm{~cm}$ (Table 1). These investigational device exemption trials have demonstrated the superiority of BMS over balloon angioplasty in longer lesions. This is due primarily to a reduction in elastic recoil and elimination of late lumen loss (LLL) secondary to constrictive remodeling. For drug delivery technologies to have a cost-effective and clinically meaningful benefit, they must provide equivalent or superior patency rates in more challenging anatomy. For example, a drug-eluting platform that increases the primary patency of a relatively short SFA lesion is of far less value than one that would render the same patency in the popliteal artery. It is likely that the future of peripheral drugeluting technology will exist on a variety of platforms that are best suited for differing vascular territories.

\section{Challenges of the SFA}

The unique anatomic, hemodynamic, and biomechanical properties of the SFA present significant challenges to both stent- and balloon-based local drug delivery. Movements necessary for activities of daily living as simple as walking exert mechanical forces which can result in material fatigue and fractures of stents placed within the SFA [1-3]. 
Table 1 Summary of trial characteristics

\begin{tabular}{|c|c|c|c|c|c|c|c|c|}
\hline Trial & Device & $\begin{array}{l}\text { Sample } \\
\text { size }\end{array}$ & $\begin{array}{l}\text { Rutherford } \\
\text { category } \\
\leq 3 />3 \\
(\%)\end{array}$ & $\begin{array}{l}\mathrm{DM} \\
(\%)\end{array}$ & $\begin{array}{l}\text { Average } \\
\text { lesion } \\
\text { length } \\
(\mathrm{cm})\end{array}$ & $\begin{array}{l}\text { Stent } \\
\text { fracture } \\
(\%)\end{array}$ & $\begin{array}{l}\text { Occlusions } \\
(\%)\end{array}$ & $\begin{array}{l}\text { Primary } \\
\text { end point }\end{array}$ \\
\hline \multicolumn{9}{|l|}{ BMS trials } \\
\hline Absolute & Dynalink vs PTA & 51 & $88 / 12$ & 43 & $10.1 \pm 7.5$ & 2 & 37 & 12-m binary restenosis \\
\hline FAST & Bard Luminexx 3 vs PTA & 123 & $97.5 / 2.5$ & 35.8 & $4.5 \pm 2.8$ & 12 & 36.6 & 12-m binary restenosis \\
\hline ASTRON & Astron vs PTA & 34 & $91 / 9$ & 29 & $8.2 \pm 6.7$ & NR & 38 & $12 \mathrm{~m}$ binary restenosis \\
\hline RESILIENT & Lifestent vs PTA & 134 & $100 / 0$ & 38 & $7.1 \pm 4.3$ & 3.1 & 17 & 12-m TLR \\
\hline SUPER & S.M.A.R.T. v PTA & 74 & $89 / 11$ & 23 & $12.3 \pm 5.4$ & NR & 95.9 & $12-\mathrm{m}$ binary restenosis \\
\hline DURABILITY I & Everflex & 151 & $87.4 / 12.6$ & 45.7 & $9.6 \pm 2.7$ & 8.1 & 40 & 12-m binary restenosis \\
\hline DURABILITY II $^{\mathrm{a}}$ & Everflex & 287 & $95 / 5$ & 43 & 8.9 & 0.4 & 48 & $12-\mathrm{m}$ primary patency \\
\hline STROLL $^{\mathrm{a}}$ & S.M.A.R.T. & 250 & & 47 & 7.7 & 1.8 & 24 & \\
\hline \multicolumn{9}{|c|}{ Drug-coated-stent trials } \\
\hline SIROCCO I/II & $\begin{array}{l}\text { S.M.A.R.T. and } \\
\text { sirolimus vs BMS }\end{array}$ & 93 & NR & 43 & $8.5 \pm 4.4$ & 17 & 69 & In-stent restenosis \\
\hline Zilver PTX & Zilver PTX vs PTA & 236 & $91 / 9$ & 49.2 & $5.4 \pm 4.1$ & 0.9 & 29.6 & 12-m binary restenosis \\
\hline STRIDES & Dynalink and everolimus & 104 & $83 / 17$ & 39 & $9.0 \pm 4.3$ & 0 & 45 & Instent restenosis \\
\hline \multicolumn{9}{|c|}{ Drug-coated-balloon trials } \\
\hline THUNDER & $\begin{array}{l}\text { Paclitaxel coated vs } \\
\text { paclitaxel and contrast } \\
\text { medium vs PTA }\end{array}$ & 48 & NR & 50 & $7.5 \pm 6.2$ & NA & 27 & 6-m LLL \\
\hline FemPAC & Paclitaxel coated vs PTA & 45 & $96 / 4$ & 40 & 6.1 & NA & 13 & 6-m LLL \\
\hline PACIFIER & $\begin{array}{l}\text { Paclitaxel-coated IN.PACT } \\
\text { Pacific vs PTA }\end{array}$ & 44 & $95.5 / 4.5$ & 43.2 & $7.0 \pm 5.3$ & NA & 22.7 & 6-m LLL \\
\hline LEVANT I $^{\mathrm{a}}$ & Moxy vs PTA & 49 & $93 / 6$ & 45 & $8.1 \pm 3.7$ & NA & 40 & 6-m LLL \\
\hline
\end{tabular}

$B M S$ bare metal stent, $D M$ diabetes mellitus, $L L L$ late lumen loss, NA not applicable, NR not reported, PTA percutaneous transluminal angioplasty, TLR target lesion revascularization

${ }^{a}$ Not yet published

Micromovement of the stent on the vessel wall creates repetitive friction and inflammation. Accordingly, the SFA has been shown to respond to stent implantation with a more potent postimplantation inflammatory response than other arteries, which has been linked to restenosis [4, 5]. Multiple overlapping stents used to treat long-segment stenosis or occlusions in the SFA may cause metal-to-metal hinge points that initiate the fracture process.

The SFA is a muscular artery having a much thicker tunica media than the iliac or coronary counterparts. The human coronary artery has a thickness of about $300 \mu \mathrm{m}$, whereas the femoral artery has a thickness between 700 and 1,600 $\mu \mathrm{m}$ depending on the amount of atherosclerosis [6]. Heterogeneous fibrocalcific plaque and Mönckeberg's medial calcific sclerosis present diffusion barriers and unique challenges to uniform distribution of drugs delivered to the arterial wall by balloons and stents. Multilevel disease, popliteal and tibial intervention, and poor runoff, all commonly found in patients with critical limb ischemia, further complicate endovascular therapy [7-12].
The Current Standard: What Do We Know About Endovascular Treatment of the SFA with Angioplasty and BMS

Several randomized controlled trials have now been published comparing BMS with percutaneous transluminal angioplasty (PTA). Generally, these trials have shown stenting to be superior to PTA in longer lesions, yielding 12-month primary patency rates ranging from 52 to $81 \%$ (Fig. 1).

There have been five published clinical trials to date randomizing patients to implantation of self-expanding nitinol stents versus PTA [13-17]. In the Vienna Absolute trial (Balloon Angioplasty Versus Stenting With Nitinol Stents in the Superficial Femoral Artery) [13], 104 patients were randomized to primary nitinol stent implantation with Dynalink/Absolute stents (Guidant, Santa Clara, CA, USA) $(n=51$; mean lesion length $10.1 \pm 7.5 \mathrm{~cm})$ versus angioplasty with provisional and bailout stenting $(n=53$; lesion length $9.2 \pm 6.4 \mathrm{~cm})$. At 12 months, the 
investigators found significantly lower rates of binary restenosis determined by duplex ultrasonography in the primary stenting group ( 37 vs $63 \% ; p=0.01$ ). They observed a $2 \%$ fracture rate. In this trial the binary restenosis rate of the stent group at 6,12 , and 24 months was 24,37 , and $45.7 \%$ respectively [13, 18], indicating ongoing cellular proliferation between the first and second years following stent implantation.

The Femoral Artery Stenting Trial (FAST) [14] randomized patients to primary nitinol stenting with a single Bard Luminexx 3 stent $(n=123)$ versus PTA $(n=121)$, with a mean lesion length of $4.5 \pm 2.8$ in the stenting group. The investigators found equivalent results for the two treatment groups, with 12-month binary restenosis rates of $31.7 \%$ in the stent group and $38.6 \%$ in the PTA group ( $p=0.377)$. The observed rates of binary restenosis in the PTA arm were much lower than expected, so the trial was ultimately not powered to establish an absolute difference of $7 \%$. Thus, the indication for primary stenting of very short lesions of the SFA remains debatable. In addition, the investigators observed a much higher $12 \%$ stent fracture rate, despite the shorter lesion being treated.

The RESILIENT [17] and ASTRON [15] trials randomized patients with intermediate lesion lengths (7.1 and $8.2 \mathrm{~cm}$ respectively) to stenting versus PTA. The RESILIENT trial enrolled 206 patients with intermittent claudication and stenosis of the SFA and proximal popliteal artery. They underwent 2:1 randomization to stenting with the Edwards self-expanding nitinol Lifestent $(n=134)$ versus angioplasty $(n=72)$. Mean lesion length was $7.1 \mathrm{~cm}$ in the stenting group and $6.4 \mathrm{~cm}$ in the angioplasty group. At 12 months, freedom from target lesion revascularization (TLR) was higher in the stent group (87.3 vs $45.1 \%, p \leq 0.0001)$. TLR, the primary end point, was defined as any further percutaneous intervention or bypass surgery of the target lesion or vessel because of a return of ischemic symptoms, decrease of at least one Rutherford category, decrease in the ankle brachial index of more than 0.15 , or loss of patency as measured by angiography or duplex ultrasonography. Ultrasonographically determined primary patency [peak systolic velocity ratio (PSVR) of 2.5 or greater] at 12 months was 81.3 versus $36.7 \%$ $(p \leq 0.0001)$. There was a $40 \%$ suboptimal balloon angioplasty rate, necessitating bailout stenting. These were counted as immediate balloon failures and therefore the primary patency in the angioplasty arm was only $60 \%$ at the conclusion of the index procedure. In total, 161 patients were available for follow-up at 36 months, at which time there was no difference in survival or major adverse events [19]. Freedom from TLR continued to be significantly better in the stent group at 3 years (75.5 vs. $41.8 \%$, $p \leq 0.0001)$. Patency data and fracture rates were not ascertained at 3 years.

Similarly, the ASTRON trial [15] randomized 73 patients to primary stenting with the Biotronik Astron selfexpanding nitinol stent $(n=34)$ versus PTA $(n=39)$, with lesion lengths averaging $8.2 \pm 6.7 \mathrm{~cm}$. At 12 months, ultrasonographically determined (PSVR $\geq 2.4$ ) binary restenosis rates were $34.4 \%$ in the stent group and $61.1 \%$ in the PTA group $(p=0.028)$. Fracture rates were not reported in this trial. However, the results confirmed those seen in the Absolute trial, supporting the indication for primary self-expanding nitinol stenting in intermediatelength lesions of the SFA. This conclusion has been further solidified by positive results from the subsequent nonrandomized single-arm studies DURABILITY I/II [20] and STROLL (see Table 1).

The limitation of BMS was evidenced in the recent randomized trial of the S.M.A.R.T. stent versus balloon angioplasty in long SFA lesions: the SUPER study [16]. It
Fig. 1 Percent freedom from binary restenosis by trial: 12-month results unless otherwise indicated. Asterisks indicate 6-month results. $B M S$ bare metal stent, $D C B$ drug-coated balloon, $D E S$ drug-eluting stent

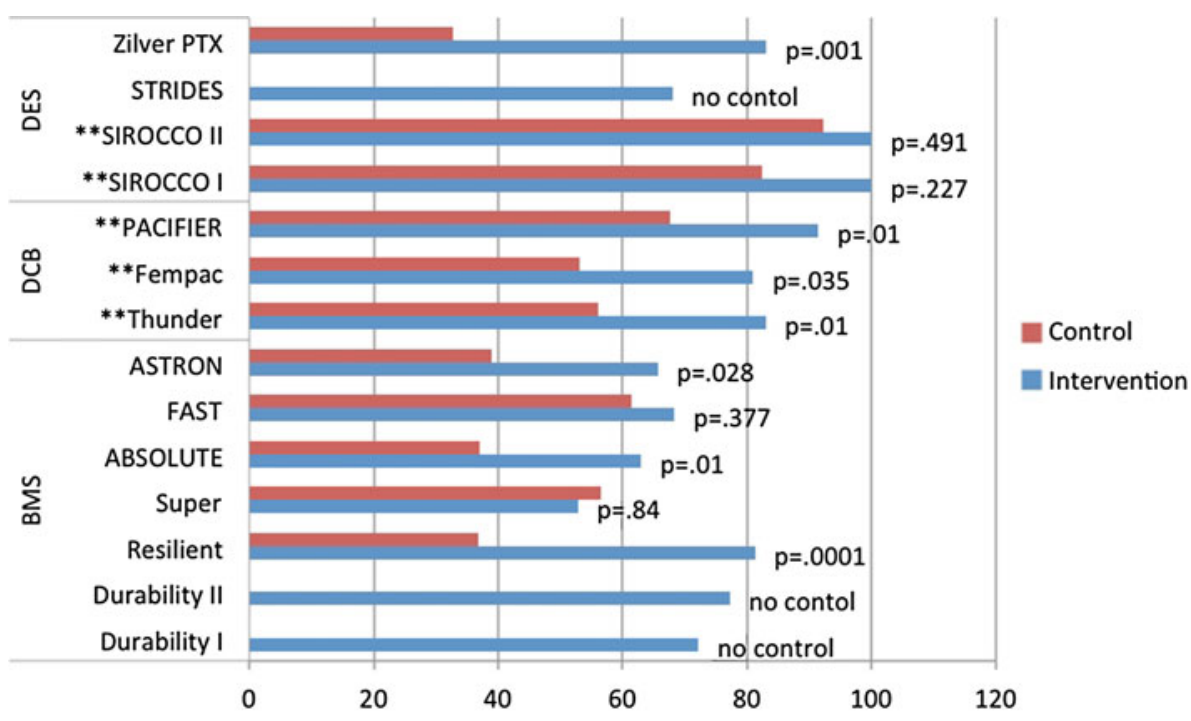


sought to extend the indications for SFA stenting by randomizing patients to primary stenting $(n=74)$ versus PTA with bailout stenting $(n=76)$. These patients had a long average lesion length of $12.3 \pm 5.4 \mathrm{~cm}$, and most of the lesions were total occlusions (95.9\%), whereas in previous trials total occlusion accounted for between 17 and $40 \%$ of the lesions. The SUPER trial found no reduction in 12-month rates of binary restenosis in this cohort of patients, with $47.2 \%$ in the primary stenting group and $43.5 \%$ in the PTA group $(p=0.84)$. This was true in intention-to-treat or as-treated analyses. This study recruited patients with lesions $20 \%$ longer and 2.5 times the number of total occlusions compared with previously published trials. As expected, it also enrolled a higher proportion of patients with critical limb ischemia. All of these factors, as previously outlined, predispose to poorer outcomes and may have reduced the expected effect size in this study. The investigators postulate that recanalization was mainly in the subintimal plane and it may be that there is less advantage of subintimal stent placement as compared with placement in the true lumen.

\section{Stent-Based Local Drug Delivery in the Femoral Artery}

The Sirolimus-Coated Cordis Self-Expandable Stent for the Treatment of SFA Disease (SIROCCO) trial was the first published study on the use of sirolimus-eluting stents in human infrainguinal vasculature $[1,2,21]$. This doubleblind, randomized controlled trial compared the efficacy of a sirolimus-coated S.M.A.R.T. CONTROL stent with that of a bare metal S.M.A.R.T. CONTROL stent in the femoropopliteal arterial segment. The sirolimus was combined with an unspecified elastic copolymer in a 30:70 drug-tocopolymer weight ratio coated to a thickness of $5 \mu \mathrm{m}$ on a $6 \mathrm{~mm} \times 80 \mathrm{~mm}$ or $7 \mathrm{~mm} \times 80 \mathrm{~mm}$ S.M.A.R.T. CONTROL nitinol self-expanding stent. The amount of drug was equivalent to that used in the coronary application, $90 \mu \mathrm{g} / \mathrm{cm}^{2}$, for a total of $1.2 \mathrm{mg}$ of sirolimus per stent. The study was performed in two phases, each with a 6-month follow-up, and each with slightly different end points.

Unfortunately, neither trial achieved a significant reduction in restenosis, and even after 4 years of follow-up there was no difference in any metric between the two treatment groups [21]. Several reasons have been suggested for this. First, the restenosis rate in the BMS arm was much lower than the estimated rate used for the power calculation and therefore the trial was underpowered to demonstrate a difference. Second, the compliant polymer employed on the stents, which was designed to allow expansion within the SFA and to accommodate biomechanical motion, may have been delaminated with repeated stress. In addition, even though the elution rate was labeled as "slow release," it was in fact faster than in the coronary arteries and therefore the retention may have been suboptimal for the therapeutic window to prevent femoral artery restenosis. Finally, the excessive stent fracture rate compromised meaningful comparisons between the two groups.

Currently, the Zilver PTX stent (Cook Medical, Bloomington, IN, USA) is the only FDA-approved drugeluting stent platform for use in the SFA available in the USA (see Table 2). Zilver PTX was a prospective, multinational trial testing the hypothesis that the Zilver PTX stent was superior to PTA alone [22••]. Cook Medical did not use a BMS as a comparator because at initiation of the trial there were no FDA-approved BMS for use in the SFA. This trial differed substantially from the SIROCCO study. First, the Zilver PTX stent uses no coating or binding polymer and therefore has no risk of delamination from repeated cyclic stress. Second, the antiproliferative agent chosen was paclitaxel loaded at a dose density of $3 \mu \mathrm{g}$ / $\mathrm{mm}^{2}$ for a maximum of $880 \mu \mathrm{g}$ of paclitaxel on the largest stent. Third, only one stent per leg was allowed, thereby reducing the risk of stent fracture, and relatively short lesions were treated, thereby potentially reducing the risk of host toxicity. The most obvious difference, however, was the choice of control arm. The Zilver PTX study used angioplasty as the control, whereas the SIROCCO study used an uncoated BMS. The trial had a primary safety end point of 12-month event-free survival defined as freedom from death, amputation, TLR, or worsening Rutherford score. The primary effectiveness end point was 12-month primary patency defined as a PSVR of less than 2.0 by duplex ultrasonography or a stenosis of less than $50 \%$ demonstrated on angiography. In total, 479 patients were enrolled in this study, 238 in the PTA arm and 241 in the Zilver PTX arm. Patients in the PTA arm who had suboptimal angioplasty could then be further randomized to implantation of either a Zilver BMS or a Zilver PTX stent. The mean lesion length was $5.4 \pm 4.1 \mathrm{~cm}$, and $29.6 \%$ of lesions were total occlusions. Primary patency at 12 months was $83.1 \%$ for the Zilver PTX stent versus $32.8 \%$ for all patients randomized to PTA, $p<0.01$. From examination of only the PTA which had an optimal result $(N=118)$, the 1-year primary patency rate was $65.3 \%$, which was still statistically inferior to that for the Zilver PTX arm $(p<0.01)$. In the subgroup undergoing secondary randomization after suboptimal PTA, the 12-month patency rate was $89.9 \%$ in the Zilver PTX group compared with $73 \%$ in the angioplasty and Zilver BMS group $(p=0.01)$. As expected in these relatively short lesions, the X-ray core laboratory identified only four stent fractures, a $0.9 \%$ fracture rate, in 12 months.

The Zilver PTX study was the largest, prospective, randomized trial for the endovascular treatment of symptomatic femoropopliteal peripheral artery disease (479 patients) presented to date, and the first human study to 
demonstrate a biologic effect of an antiproliferative agent applied to a stent-based platform in the femoral artery. Nevertheless, a criticism of the trial is that the relatively short lesions included do not represent real-world practice.

A concurrent Zilver PTX registry, however, enrolled 787 patients with mean lesion length of $9.95 \pm 8.2 \mathrm{~cm}$ [23]. Nine hundred lesions (38.3\% total occlusions, $24.3 \%$ restenoses) were treated with 1,722 Zilver PTX stents, averaging 2.2 stents per patient. With use of a conservative PSVR of 2.0 or less, $83 \%$ of the lesions were patent at 12 months; this increased to $86.2 \%$ when a PSVR of 2.5 was used.

The Superficial Femoral Artery Treatment with DrugEluting Stents (STRIDES) trial [24••] was designed to address some of the shortcomings of the Zilver PTX randomized trial by providing a higher level of drug delivery with a longer elution profile, and a stent design less prone to fatigue and fracture. This was the first clinical trial of nitinol self-expanding everolimus-eluting stents for peripheral artery disease. The drug delivery platform comprised three components: the Dynalink nitinol selfexpanding stent (Abbott Laboratories, Abbott Park, IL, USA), the antiproliferative drug everolimus, and an ethylene vinyl alcohol copolymer, collectively known as the Dynalink-E stent [25]. This nonrandomized, single-arm, multienter trial enrolled 104 patients with Rutherford scores 2-5, and de novo or restenotic occlusive lesions $3-17 \mathrm{~cm}$ in length. The stent system was designed with a relatively high drug payload $\left(225 \mu \mathrm{g} / \mathrm{cm}^{2}\right.$ everolimus) and a long elution profile (about $80 \%$ released during the first 3 months). This compares with coronary everolimus-eluting stents, which have a drug load of $100 \mathrm{ug} / \mathrm{cm}^{2}$, with $80 \%$ of the drug released over the first 30 days (see Table 3). The maximum allowable stent length per patient was $200 \mathrm{~mm}$. The primary end point of the trial was the rate of in-stent binary restenosis $(50 \%$ or more stenosis using duplex ultrasonography, PSVR $\geq 2.5$ ) at 6 months. Secondary end points included change in Rutherford score, improvement in walking capacity by walking impairment questionnaire, rates of stent fracture, primary patency, TLR, limb salvage, and survival after 12 months.

Primary patency after 6 and 12 months was $94 \pm 2.3 \%$ and $68 \pm 4.6 \%$. There were no stent fractures in 122 devices after 12 months. There were also a large number of restenotic events after the 6-month mark. This suggests that an even longer drug elution profile may be needed in longer lesions. This also begins to get at what may be the ultimate limitation of stent-based delivery platforms. Long after the drug has left the stent surface, the microfriction of the struts with the movement of the SFA may create inflammation, irritation, and ultimately restenosis. Three months after a drug-eluting stent has been placed in the SFA, one is left with a BMS in the SFA!

\section{Balloon-Based Local Drug Delivery in the Femoral Artery}

In non-stent-based drug delivery platforms where there is limited contact between the platform and the arterial surface, achieving sufficient target tissue uptake and retention while limiting systemic spillover is the most challenging problem. The drug-coated balloon (DCB) has been investigated most thoroughly in preclinical and clinical models. Similarly to the stent, the DCB must be thought of as three separate components: the platform (balloon), the drug (usually paclitaxel), and a drug carrier or spacer. Theoretically, DCB angioplasty has a number of potential advantages over standard angioplasty and stent technologies. For

Table 2 Summary of drug-eluting stents currently available in the USA

\begin{tabular}{|c|c|c|c|c|c|}
\hline Manufacturer & Device & Drug & Polymer & Indication & Trials \\
\hline $\begin{array}{l}\text { Johnson \& Johnson and } \\
\text { Cordis }\end{array}$ & Cypher & Sirolimus & 3-layer coating: Parylene C, PEVA, PBMA & Cardiac & $\begin{array}{l}\text { RAVEL, SAPPHIRE, } \\
\text { SIRIUS }\end{array}$ \\
\hline Boston Scientific & Taxus & Paclitaxel & $\begin{array}{l}\text { Translute SIBS (nonresorbable } \\
\text { elastomeric) }\end{array}$ & Cardiac & $\begin{array}{l}\text { ELUTES, TAXUS II, } \\
\text { ASPECT }\end{array}$ \\
\hline Boston Scientific & Ion & Paclitaxel & $\begin{array}{l}\text { Triblock copolymer (polystyrene and } \\
\text { polyisobutylene) }\end{array}$ & Cardiac & PERSEUS \\
\hline Boston Scientific & Promus & Everolimus & PBMA, PVDF-HFP & Cardiac & SPIRIT \\
\hline $\begin{array}{l}\text { Guidant and Abbott } \\
\text { Laboratories }\end{array}$ & Xience V & Everolimus & Fluoropolymer & Cardiac & SPIRIT \\
\hline $\begin{array}{l}\text { Guidant and Abbott } \\
\text { Laboratories }\end{array}$ & $\begin{array}{l}\text { Xience } \\
\text { Prime }\end{array}$ & Everolimus & Fluoropolymer & Cardiac & SPIRIT \\
\hline Medtronic & Endeavor & Zotarolimus & Phosphorylcholine & Cardiac & ENDEAVOR \\
\hline Cook Medical & $\begin{array}{r}\text { Zilver } \\
\text { PTX }\end{array}$ & Paclitaxel & None & Femoropopliteal & Zilver PTX \\
\hline
\end{tabular}

HFP hexafluoropropylene, $P B M A$ poly(n-butyl methacrylate), $P E V A$ poly(ethylene-co-vinyl acetate), $P V D F$ poly(vinylidene difluoride), SIBS poly(styrene- $b$-isobutylene- $b$-styrene) 
Table 3 Drug concentrations of everolimus-eluting stents

\begin{tabular}{lc}
\hline Stent & Everoimus dose $\left(\mu / \mathrm{cm}^{2}\right)$ \\
\hline Xience V & 100 \\
Cypher & 140 \\
SIROCCO & 90 \\
Dynalink-E & 225 \\
\hline
\end{tabular}

example, there is a potential for homogenous drug delivery to the vessel wall, which is not accomplished with the concentration gradients produced by drug-eluting stents. There is immediate drug release without the use of a polymer that can induce chronic inflammation and late thrombosis as observed with some drug-eluting stents. Therefore, no foreign object is left behind. A DCB may be useful in sections of the femoral artery where it would be undesirable to place a stent such as the common and profunda femoral artery or the popliteal artery. Finally, without the presence of a polymer-coated stent, there is potential for reducing antiplatelet therapy.

However, several safety considerations merit close attention. The percentage of drug absorption into the tissue versus that released into the bloodstream versus that which remains on the balloon must be considered. Redistribution of drug from the arterial wall into distal musculature, spleen, and other organs may cause unwanted side effects. Balloons are inherently a less efficient delivery mechanism than stents and could have greater systemic toxicity. Information on how the balloon was coated and how it maintains consistency and uniformity across a range of sizes is important. Fibrocalcific plaque, which is common to the SFA, dissection of the artery from balloon angioplasty, and turbulence produce unpredictable drug uptake and patchy delivery into the arterial wall. Finally DCB technology, similar to conventional balloon angioplasty, cannot prevent early elastic recoil and LLL due to negative remodeling.

Two German multicenter clinical DCB studies have been published, both using the same coating technology and the same drug, and yielded very similar results. The first human examination of DCB in the femoral artery, the Local Taxane with Short Exposure for Reduction of Restenosis in Distal Arteries (THUNDER) trial [26], was a multicenter study that involved a three-way randomization of 154 patients with either stenosis or occlusion of a femoropopliteal segment comparing standard balloon angioplasty (control) with an iopromide-paclitaxel $(3 \mu \mathrm{g} /$ $\mathrm{mm}^{2}$ ), Cotavance DCB (Paccocath technology, MEDRAD, an affiliate of Bayer HealthCare) or with intra-arterial injection of paclitaxel mixed with iopromide contrast medium. In total, 48 patients were treated with the DCB. There was a 60 -s inflation time for the study balloons. For patients treated with the coated balloons, the mean dose of paclitaxel per patient was $5 \mathrm{mg}$ (range $1-17 \mathrm{mg}$ ); for those treated with paclitaxel added to contrast medium, the mean dose was $17 \mathrm{mg}$. With a mean lesion length of $7.5 \mathrm{~cm}$, there was marked reduction in the iopromide-paclitaxel balloon group for the primary end point of 6-month angiographic LLL compared with both the control balloon group and the paclitaxel in contrast medium group $(0.4 \pm 1.2$ vs $1.7 \pm 1.8$ vs $2.2 \pm 1.6 \mathrm{~mm} ; p<0.001$ for DCB versus control). Similarly the angiographic restenosis rates were significantly lower among patients treated with the paclitaxel-coated balloons compared with control balloons (17 vs. $44 \%, p=0.01)$. These patients have now been followed up to 12,24 , and 60 months (60-month data reported but not published). Only clinical assessments were reported for 12 and 24 months. TLR at 12 months was noted to be $10 \%$ in the DCB group compared with 48 and $35 \%$ in the control and intra-arterial paclitaxel groups, respectively $(p<0.001)$. Clinically driven TLR remained statistically significant at 24 months, $15 \%$ in the DCB group versus 52 and $40 \%$ in the other two groups $(p<0.001)$ [26]. Twenty-five patients from the DCB group and 21 from the PTA group were available for follow-up at 5 years. There was no longer a difference in LLL for these patients $(0.7 \pm 1.9$ vs $1.5 \pm 1.3 \mathrm{~mm} ; p=0.54)$. TLR was $21 \%$ for the DCB group and $56 \%$ for the PTA group $(p<0.001)$ [27].

In the Femoral Paclitaxel (FemPac) trial [28], 87 patients underwent 1:1 randomization between control balloon angioplasty and iopromide-paclitaxel-coated balloon angioplasty (Cotavance balloon with Paccocath technology, MEDRAD, Bayer HealthCare) in relatively short $(6-\mathrm{cm})$ lesions in the femoropopliteal arteries. In this study a mean of $3.7 \mathrm{mg}$ (range $1.3-12.2 \mathrm{mg}$ ) of paclitaxel was delivered per patient. The coated balloon exhibited significantly less LLL (primary end point) at 6 months than the control balloon $(0.5 \pm 1.1$ vs $1.0 \pm 1.1 \mathrm{~mm}$, $p=0.031)$ and angiographic restenosis was significantly lower in the treated group than the control group (19 vs $47 \% ; p=0.035$ ).

Hence, both the THUNDER trial and the FemPac trial demonstrated proof-of-concept that a short-term exposure of injured femoral arteries to paclitaxel may be sufficient to inhibit restenosis. However, as with any proof-of-concept trial, both investigations must be considered within the context of their limitations: small sample sizes, unconventional surrogate end points, heterogeneous patient population, incomplete follow-up, incomplete blinding, and only a short-term angiographic follow-up. In the THUNDER trial, $6.6 \%$ of the paclitaxel and coating residue and in the FemPac trial, $6.4 \%$ of the paclitaxel and coating residue remained on the balloon after intervention. It is not known how much of the intended payload was delivered to the vessel wall. Determination of nontargeted drug delivery to 
the bloodstream and other tissue is an important safety concern, especially when treating longer, more complicated lesions that potentially require multiple inflations. Clearly longer follow-up is needed to determine if the angiographic efficacy seen in these studies can be sustained and translated into clinically meaningful end points such as patency rates and improved walking distances.

The Paclitaxel-Coated Balloons in Femoral Indication to Defeat Restenosis (PACIFIER) trial [29••] enrolled 91 patients with an average lesion length of $7.0 \pm 5.3 \mathrm{~cm}$ (range $3-30 \mathrm{~cm}$ ). Forty-four patients were randomized to treatment with a paclitaxel-coated IN.PACT Pacific balloon (Medtronic), and 47 were randomized to standard balloon angioplasty with provisional stenting. Thirty percent of patients had total occlusions. The IN.PACT Pacific is a balloon coated with paclitaxel $\left(3 \mu \mathrm{g} / \mathrm{mm}^{2}\right)$ and urea as a hydrophilic natural spacer. The total dose of paclitaxel in the DCB group ranged from 1.5 to $21.0 \mathrm{mg}$ per intervention and $86 \pm 12 \%$ of the dose was released from the balloons. The primary end point of this trial was angiographic LLL at 6 months. The DCB group had significantly better LLL $(0.01$ vs $0.65 \mathrm{~mm} ; p=0.0014)$, as well as a significantly better binary restenosis rate ( 8.6 vs $32.4 \% ; p=0.01)$ at 6 months. At 12 months, there were fewer clinically driven TLRs in the DCB group verses the noncoated balloon control group (7.1 vs $27.9 \%, p=0.02$ ). Again, however, this trial suffers from small sample size and short follow-up times and no mention of systemic drug levels.

The LEVANT I study [30] is a German/Belgium prospective, multicenter, single blind (subject), randomized controlled trial comparing the Lutonix (Moxy) paclitaxelcoated balloon catheter versus standard balloon angioplasty for treatment of femoropopliteal lesions with and without stenting. The trial is completed but as yet the results have not been published. The analysis involved 49 patients in the Moxy arm and 52 patients in the standard treatment arm. Moxy is a drug-eluting balloon coated with $2 \mu \mathrm{g} / \mathrm{mm}^{2}$ paclitaxel with a hydrophilic nonpolymer polysorbate and sorbitol carrier. The drug is delivered during a single 30-s inflation. The primary efficacy end point for this study was LLL at 6 months. The investigators included patients with Rutherford category 2-5 disease and lesion lengths from 4 to $15 \mathrm{~cm}$ (average $8.1 \mathrm{~cm}$ ). In the intention-to-treat analysis, the Moxy balloon yielded a 0.46-mm LLL compared with a 1.09-mm LLL with the standard balloon $(p=0.016)$. LEVANT II is an extension of this trial with ongoing enrollment of 700 patients. The primary end point is primary patency at 12 months as well as composite end point of freedom from all-cause perioperative ( 30 days oe less) death and freedom from the following: index limb amputation, index limb reintervention, and index-limb-related death, all at 12 months. The LEVANT investigators have not disclosed details of the carrier molecule or how much paclitaxel is spilled into the circulation during delivery and inflation of the balloon.

It must be noted that relatively few patients have been treated with DCBs. The trials reviewed here and published to date involve only 186 patients, most with only 6-month follow-up. So although the initial results are promising, we must await further trials with longer follow-up and more traditional end points. Many trials are ongoing both in Europe (FREERIDE, ADVANCE 18 PTX, ISAR-STATH, FAIR, COPA CABANA, ISAR-PEBIS) and in the USA (LEVANT II, INPACT SFA II).

\section{Conclusions}

Innovative local drug delivery platforms are likely to improve on existing endovascular therapy in the periphery. Several proof-of-concept trials have either been published or been recently presented and many more are in the pipeline suggesting biologic effectiveness of these hybrid devices in reducing restenosis. This technology has already been commercially introduced in some countries for a variety of clinical settings. However, caution and safety are paramount. Adequately powered, multicenter, welldesigned, randomized controlled trials with long-term follow-up (3-5 years) are needed to accurately assess safety and efficacy.

Disclosure Joy P. Walker declares that she has no conflict of interest. Christopher D. Owens declares that he has no conflict of interest.

\section{References}

Papers of particular interest, published recently, have been highlighted as:

•- Of major importance

1. Duda $\mathrm{SH}$, et al. Sirolimus-eluting versus bare nitinol stent for obstructive superficial femoral artery disease: the SIROCCO II trial. J Vasc Interv Radiol. 2005;16(3):331-8.

2. Duda SH, et al. Sirolimus-eluting stents for the treatment of obstructive superficial femoral artery disease: six-month results. Circulation. 2002;106(12):1505-9.

3. Scheinert D, et al. Prevalence and clinical impact of stent fractures after femoropopliteal stenting. J Am Coll Cardiol. 2005;45(2): $312-5$.

4. Schillinger $\mathrm{M}$, et al. Balloon angioplasty and stent implantation induce a vascular inflammatory reaction. J Endovasc Ther. 2002;9(1): 59-66.

5. Schillinger $\mathrm{M}$, et al. Inflammatory response to stent implantation: differences in femoropopliteal, iliac, and carotid arteries. Radiology. 2002;224(2):529-35.

6. Smilde TJ, et al. Carotid and femoral artery wall thickness and stiffness in patients at risk for cardiovascular disease, with special emphasis on hyperhomocysteinemia. Arterioscler Thromb Vasc Biol. 1998;18(12):1958-63. 
7. DeRubertis BG, et al. Lesion severity and treatment complexity are associated with outcome after percutaneous infra-inguinal intervention. J Vasc Surg. 2007;46(4):709-16.

8. Surowiec SM, et al. Percutaneous angioplasty and stenting of the superficial femoral artery. J Vasc Surg. 2005;41(2):269-78.

9. Kudo T, Chandra FA, Ahn SS. Long-term outcomes and predictors of iliac angioplasty with selective stenting. J Vasc Surg. 2005;42(3):466-75.

10. Black JH 3rd, et al. Contemporary results of angioplasty-based infrainguinal percutaneous interventions. J Vasc Surg. 2005; 42(5):932-9.

11. Conrad MF, et al. Intermediate results of percutaneous endovascular therapy of femoropopliteal occlusive disease: a contemporary series. J Vasc Surg. 2006;44(4):762-9.

12. Samson RH, et al. CryoPlasty therapy of the superficial femoral and popliteal arteries: a single center experience. Vasc Endovasc Surg. 2006;40(6):446-50.

13. Schillinger $M$, et al. Balloon angioplasty versus implantation of nitinol stents in the superficial femoral artery. $\mathrm{N}$ Engl $\mathrm{J}$ Med. 2006;354(18):1879-88.

14. Krankenberg $\mathrm{H}$, et al. Nitinol stent implantation versus percutaneous transluminal angioplasty in superficial femoral artery lesions up to $10 \mathrm{~cm}$ in length: the femoral artery stenting trial (FAST). Circulation. 2007;116(3):285-92.

15. Dick $\mathrm{P}$, et al. Balloon angioplasty versus stenting with nitinol stents in intermediate length superficial femoral artery lesions. Catheter Cardiovasc Interv. 2009;74(7):1090-5.

16. Chalmers N, et al. Randomized trial of the SMART stent versus balloon angioplasty in long superficial femoral artery lesions: the SUPER study. Cardiovasc Intervent Radiol. 2013;36(2):353-61.

17. Laird JR, et al. Nitinol stent implantation versus balloon angioplasty for lesions in the superficial femoral artery and proximal popliteal artery: twelve-month results from the RESILIENT randomized trial. Circ Cardiovasc Interv. 2010;3(3):267-76.

18. Schillinger $M$, et al. Sustained benefit at 2 years of primary femoropopliteal stenting compared with balloon angioplasty with optional stenting. Circulation. 2007;115(21):2745-9.

19. Laird JR, et al. Nitinol stent implantation vs. balloon angioplasty for lesions in the superficial femoral and proximal popliteal arteries of patients with claudication: three-year follow-up from the RESILIENT randomized trial. J Endovasc Ther. 2012;19(1):1-9.

20. Bosiers M, et al. Nitinol stent implantation in long superficial femoral artery lesions: 12-month results of the DURABILITY I study. J Endovasc Ther. 2009;16(3):261-9.

21. Duda SH, et al. Drug-eluting and bare nitinol stents for the treatment of atherosclerotic lesions in the superficial femoral artery: long-term results from the SIROCCO trial. J Endovasc Ther. 2006;13(6):701-10.

22. •• Dake MD, et al. Paclitaxel-eluting stents show superiority to balloon angioplasty and bare metal stents in femoropopliteal disease: twelve-month Zilver PTX randomized study results. Circ Cardiovasc Interv. 2011;4(5):495-504. The Zilver PTX study was the largest, prospective, randomized trial of drug-eluting stents for the endovascular treatment of symptomatic femoropopliteal peripheral artery disease (479 patients) and the first human study to demonstrate a biologic effect of an antiproliferative agent applied to a stent-based platform in the femoral artery. A criticism of the trial is that the relatively short lesions included do not represent real-world practice.

23. Dake MD, et al. Nitinol stents with polymer-free paclitaxel coating for lesions in the superficial femoral and popliteal arteries above the knee: twelve-month safety and effectiveness results from the Zilver PTX single-arm clinical study. J Endovasc Ther. 2011;18(5):613-23.

24. • Lammer J, et al. First clinical trial of nitinol self-expanding everolimus-eluting stent implantation for peripheral arterial occlusive disease. J Vasc Surg. 2011;54(2):394-401. This nonrandomized, single-arm study was the first clinical trial of nitinol self-expanding everolimus-eluting stents for peripheral artery disease. Primary patency after 6 and 12 months was $94 \pm 2.3 \%$ and $68 \pm 4.6 \%$. There were a large number of restenotic events after the 6-month mark, suggesting that an even longer drug elution profile may be needed in longer lesions.

25. Lammer J, et al. Pharmacokinetic analysis after implantation of everolimus-eluting self-expanding stents in the peripheral vasculature. J Vasc Surg. 2012;55(2):400-5.

26. Tepe G, et al. Local delivery of paclitaxel to inhibit restenosis during angioplasty of the leg. N Engl J Med. 2008;358(7):68999.

27. Gunnar Tepe TZ, Albrecht T, et al. 5-year THUNDER follow-up: patients with PAD treated with uncoated versus paccocath paclitaxel coated balloons. In: Transcatheter cardiovascular therapeutics (TCT) scientific symposium; 2011; San Francisco.

28. Werk M, et al. Inhibition of restenosis in femoropopliteal arteries: paclitaxel-coated versus uncoated balloon: femoral paclitaxel randomized pilot trial. Circulation. 2008;118(13):1358-65.

29. • Werk M, et al. Paclitaxel-coated balloons reduce restenosis after femoro-popliteal angioplasty: evidence from the randomized PACIFIER trial. Circ Cardiovasc Interv. 2012;5(6):831-40. This randomized controlled trial of paclitaxel-coated IN.PACT Pacific balloons showed significantly better LLL as well as a significantly better binary restenosis rate at 6 months in the DCB group. At 12 months, there were fewer clinically driven TLRs in the DCB group verses the noncoated balloon control group. This trial suffers from small sample size and short follow-up times and no mention of systemic drug levels.

30. Scheinert D. 6 month results of the LEVANT I trial: a comparison of the Moxy ${ }^{\mathrm{TM}}$ drug coated balloon catheter vs. standard PTA for femoropopliteal disease. 2010. 